Portland State University

PDXScholar

$12-2018$

\title{
Modeling Harmonic Impacts of Electric Vehicle Chargers on Distribution Networks
}

Nicole Woodman

Intel Corporation

Robert B. Bass

Portland State University, robert.bass@pdx.edu

Mike Donnelly

A Siemens Business

Follow this and additional works at: https://pdxscholar.library.pdx.edu/ece_fac

Part of the Electrical and Computer Engineering Commons

Let us know how access to this document benefits you.

\section{Citation Details}

N. Woodman, R. B. Bass and M. Donnelly, "Modeling Harmonic Impacts of Electric Vehicle Chargers on Distribution Networks," 2018 IEEE Energy Conversion Congress and Exposition (ECCE), Portland, OR, 2018, pp. 2774-2781. doi: 10.1109/ECCE.2018.8558207

This Post-Print is brought to you for free and open access. It has been accepted for inclusion in Electrical and Computer Engineering Faculty Publications and Presentations by an authorized administrator of PDXScholar. Please contact us if we can make this document more accessible: pdxscholar@pdx.edu. 


\section{Modeling Harmonic Impacts of Electric Vehicle Chargers on Distribution Networks}

\author{
Nicole Woodman \\ Intel Corporation \\ Hillsboro, Oregon, USA \\ nicole.p.woodman@intel.com
}

\author{
Robert B. Bass \\ Portland State University \\ Portland, Oregon, USA \\ robert.bass@pdx.edu
}

\author{
Mike Donnelly \\ Mentor, A Siemens Business \\ Wilsonville, Oregon, USA \\ mike_donnelly@mentor.com
}

\begin{abstract}
As the proliferation of electric vehicles continues to grow, it is becoming important to understand the impacts that electric vehicle charging will have on distribution assets. EV chargers are non-linear, multi-state loads. This manuscript presents a design method for the modeling of $\mathrm{EV}$ charging units using a VHDL-AMS simulation environment, per IEEE Standard 1076.1. Voltage and current data collected from in-service EV charging stations were used to create harmonic profiles of the $\mathrm{EV}$ charging units. From these profiles, generalized models for both Level 2 and Level $3 \mathrm{EV}$ chargers were created. These models were validated within a larger system context using the IEEE 13 node test feeder. A VHDL-AMS tool has been created so distribution engineers may assess the impacts that EV chargers have on distribution assets. The tool may also be used to assist with the selection of transformers, conductors, and protection equipment.
\end{abstract}

Keywords-Electric vehicle charging, harmonics, system imbalance, TDD, VHDL-AMS.

\section{INTRODUCTION}

Electric vehicle (EV) chargers are a new and atypical load that power distribution systems must accommodate [1]. According to the U.S. Department of Energy's Alternative Fuels Data Center, the deployment of public electric vehicle (EV) charging stations has increased dramatically in recent years. As of Q2 2018, there are over 18,300 EV charging stations installed within the United States [2]. With the continued release of new consumer EVs to the market, this trend only shows signs of increasing.

Non-linear loads, such as EV chargers, may induce power quality (PQ) issues within distribution feeders, which in turn could shorten asset lifetimes. PQ is a measure of the fitness of electrical power provided by a utility to its customers. PQ is of concern because it manifests as deviations in voltage magnitude, issues with continuity of service from the utility, or transient voltages and currents [3]. PQ also encompasses harmonic distortion, DC offset, phase imbalance, and voltage deviations. Of special interest are harmonic currents since these have the potential to affect the lifetime of magnetic assets such as distribution transformers and instrument transformers.

Distribution engineers need models that represent the multi-state, unbalanced, and distorted nature of these loads in order to predict the impacts that EV chargers may have on distribution assets. We have developed a modeling tool that can be used to aid design of distribution feeders subject to EV loads, which are non-linear, multi-state, and unbalanced [4].
This tool can be used to observe the harmonic content of current within a feeder containing multiple EV loads; for longterm asset planning to predict asset lifetimes; and, to upgrade existing assets as EV loads are added to a feeder. By modeling these high-harmonic loads, engineers can design distribution systems to accommodate large-scale adoption of EVs.

In our previous work, we introduced a VHDL-AMS modeling environment for nonlinear, time-varying electric vehicle loads [5]. In this paper, we model Level 2 and Level 3 EV chargers within the IEEE 13 node distribution test feeder in order to observe the resulting harmonic distortion that occurs. The objectives of this work are twofold. One: to develop a tool that provides distribution engineers with a platform on which to create system designs that take into consideration the harmonic effects of EV chargers. And, two: to expand the electric utility industry's understanding of the issues that electric vehicles cause to distribution systems, thereby enabling them to protect distribution assets.

\section{BACKGROUND}

Utilities manage assets by anticipating the nature of loads and selecting assets designed to handle those loads. Modeling provides a means for understanding issues such as PQ that are specific to EVs. Such models aid in the design of distribution systems, and provide guidance for asset planning. PQ particularly affects magnetic assets, so understanding the PQ of non-linear loads can assist distribution engineers with selection of k-factor ratings for distribution transformers, and selection of current transformers (CT) and potential transformers (PT). PQ also affects protection settings and decisions regarding conductor ampacity [6].

Harmonic distortion is a deviation of a current or voltage waveform from a perfect sinusoidal profile. The impacts that harmonic distortion have on distribution assets can be detrimental to the proper operation of power systems. Harmonic distortion can also affect near-by loads, particularly power electronics devices and motors. In the case of non-linear loads, such as EV charge controllers, current distortion occurs due to the use of power electronics switches to convert power from AC to DC. Introduction of harmonic currents into the distribution system can distort the utility supply voltage and damage electrical distribution equipment, particularly magnetic components.

In order to help mitigate these adverse affects, the IEEE established Standard 519-1992, with the objective of developing 
"recommended practices and requirements for harmonic control in electrical power systems" [7]. This standard describes the problems that unmitigated harmonic current distortion can cause within electrical systems as well as the degree to which harmonics can be tolerated by a given system. The standard recognizes the responsibility of an electrical user to not degrade the voltage of the utility by drawing heavy, distorted currents.

\section{A. PQ Affects on Power System Equipment}

1) Transformers: One example of transformer losses caused by high harmonic content is $\mathrm{I}^{2} \mathrm{R}$ losses, which are due to higher-order currents within the transformer windings. If the root mean square value of the load current is increased due to a harmonic component, the $\mathrm{I}^{2} \mathrm{R}$ losses increase accordingly [8]. Consequently, the transformer will consume more real power than anticipated, reducing its efficiency.

Harmonics also induce additional eddy current and hysteresis losses in transformer cores. These losses cause abnormal temperature rise in the transformer, which accelerates the degradation of transformer insulation, ultimately leading to a shortened life span for the equipment [9]. Hysteresis and eddy current losses are frequency dependent, proportional to $f$ and $f^{2}$, respectively. As such, higher order harmonics are particularly problematic for transformers.

Stray flux is another source of losses within transformers. These occur in the core, clamps, tank and other ferric components of the transformer. Stray flux induces eddy current and hysteresis losses in these components, leading to increases in oil temperature and hot spot temperatures within the transformer. This can also contribute to the premature degradation of the transformer insulation and oil, leading eventually to equipment failure.

2) Power Cables: The primary effect of harmonics on power cables is the additional heating due to an increase in $\mathrm{I}^{2} \mathrm{R}$ losses. The increase in power dissipation across a conductor is attributed to two phenomena: the proximity effect, which results in current crowding; and, the skin effect, which forces electric current to the outer surfaces of the line. Both of these cause an effective increase in the resistance of the line that is proportional to the frequencies of the harmonic components. Also, cables involved in system resonance may be subjected to voltage stress and corona, which can lead to dielectric (insulation) failure.

3) Relays, Switch Gear, and Metering Equipment: Protective relaying equipment, switch gear, and metering equipment may also be negatively impacted by the presence of harmonic currents. Relaying equipment may operate more slowly because of higher pick-up values than settings would otherwise dictate, resulting in unexpected operation. Fuses may experience premature operation due to $I^{2} R$ heating by harmonics. And as with power transformers, harmonic currents can increase heating in CTs and VTs due to $\mathrm{I}^{2} \mathrm{R}$, eddy currents and core saturation, leading to shortened asset lifetimes. Within switchgear, the presence of harmonics contributes to $\mathrm{I}^{2} \mathrm{R}$ heating, reduces steady-state ampacity, and shortens lifetimes of insulating components.
4) Capacitors: Harmonics introduced by non-linear loads may interact with nearby capacitors if the harmonic frequency is in resonance with an LC time constant. The inherent negative reactance of conductors, transformers and loads can couple with the positive reactance of capacitor banks, resulting in very high voltages and currents at resonant frequencies. The unexpected increased voltage stress and $\mathrm{I}^{2} \mathrm{R}$ heating within resonating capacitors can result in a shortened asset lifetime or catastrophic failure.

\section{B. Triplen Currents}

Non-linear loads can induce triplen currents within threephase systems. Triplen currents comprise the non-even multiples of $3 n$, known as the triplen harmonics [10]. Examples of these are the $3^{r d}, 9^{t h}$ and $15^{t h}$ harmonics. Triplen currents are troublesome because they sum in the neutral line of grounded wye-configured systems. When these triplen currents superpose in the neutral line, they can cause excessive currents and can lead to conductor heating [11]. Similarly, triplen currents circulate within delta-wired system, which must be oversized to accommodate these currents.

\section{System Imbalance}

When system imbalance occurs, the current and voltage in one phase differs from that in another. This can cause zero and negative sequence components to arise. These currents are a measure of the imbalance in polyphase circuits. These currents can cause loading on the phases and neutral line greater that exceed design limits. Single-phase, two-wire chargers connected to a common service present imbalance problems because chargers come online in a stochastic manner. When one phase is heavily loaded, another will be loaded more lightly.

IEEE Standard 1459-2010 defines methods for calculating electric power under unbalanced conditions [12]. The standard replaces the simple apparent power calculation, $S_{e}=V I^{*}$, with an effective apparent power definition that quantifies apparent power in a manner that accommodates unbalanced loading.

IEEE 1459-2010 quantifies the active and reactive powers in a three-phase unbalanced system, as illustrated by the Power Resolution Tree, shown in Figure 1 [13]. The standard breaks down the definition of effective apparent power for the system, $S_{e}$, into fundamental and non-fundamental components ( $S_{e 1}$ and $S_{e N}$, respectively), positive sequence components $\left(S_{1}^{+}, P_{1}^{+}\right.$and $\left.Q_{1}^{+}\right)$and system imbalance as quantified by fundamental imbalance power $S_{1 u}$. Finally, harmonic active $\left(S_{e H}, P_{e H}\right.$ and $\left.D_{e H}\right)$ and distortion $\left(D_{e I}\right.$ and $\left.D_{e V}\right)$ powers are described as well. Cataliotti et al. demonstrate the value of using IEEE 1459-2010 for quantifying harmonic content [14].

\section{Harmonic Distortion}

EVs employ power electronics within the charge controllers that interface the vehicle's electric power system with the grid. For Level 1 and Level 2 chargers, vehicle charging is done by an on-board AC-DC controlled rectifier that couples with the electric service via a single phase connector. For Level 3 chargers (DC Fast Chargers) the charging is controlled by electronics within the charge controller [15]. 


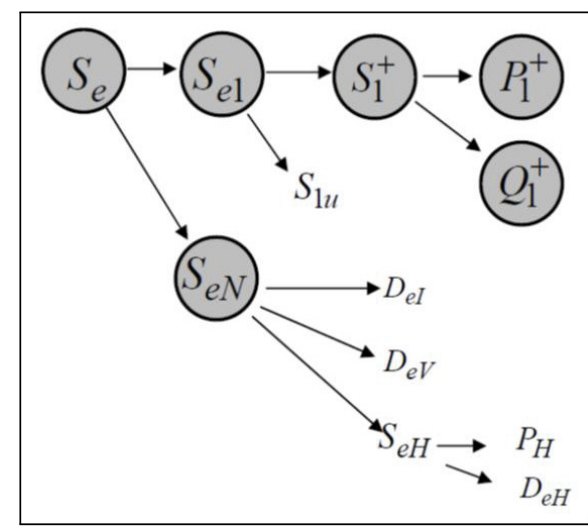

Fig. 1: The power resolution tree for three-phase non-sinusoidal conditions. [13]

1) Total Harmonic Distortion: The harmonic distortion introduced into the distribution system by charge controllers can be measured in terms of Total Harmonic Distortion, THD. IEEE Standard 519-1992 recommends limits for THD of loads within a distribution system [7]. However, the THD of a charger changes throughout the charging cycle as the firing angles of the power electronics switches change in response to the various states of the charging cycle. Further, the THD on a utility feeder is compounded when multiple EVs are connected to the same service [16], [17]. Equation 1 shows the mathematical definition of THD. Note THD is a ratio of a load's harmonic current content with respect to the load's fundamental current, $I_{1}$; THD is a metric of the harmonic content of a single load, and not a metric of the harmonic content within a feeder.

$$
T H D=\frac{\sqrt{\sum_{n=2}^{\infty} I_{n}^{2}}}{I_{1}}
$$

2) Total Demand Distortion: Total Demand Distortion (TDD) considers distortion of all the loads on a feeder with respect to the size of the distribution feeder. The maximum allowable TDD is determined by the ratio of the short circuit current at the point of common coupling to the average maximum demand load current for the feeder for the previous 12 months, $I_{L}$ [18]. As defined by IEEE Standard 519-1992, TDD quantifies the percentage of harmonic current that may be inject onto a feeder [7]. As the size of the distorted load increases with respect to the feeder size, the percentage of harmonic current injection is lowered. This is a challenge for EV loads. EV charging draws high current, and the TDD changes throughout a charging event. Ideally, the harmonic distortion caused by a single consumer should be limited to an acceptable level at all points along the feeder. The prescribed levels for TDD establish the maximum allowable current distortion for a given system [7]. Equation 2 shows the mathematical definition of TDD.

$$
T D D=\frac{\sqrt{\sum_{n=2}^{\infty} I_{n}^{2}}}{I_{L}}
$$

\section{EV CHARger MODELS}

The load behavior of EV charging units is problematic for power systems and the components associated with them [19][21]. While there have been various attempts to model these chargers, each charger design is different from the next and usually contains some sort of proprietary design, making the modeling task more than trivial [22]-[24]. There are certain aspects of the characterization of these chargers that have proven difficult to present in a generalized fashion that is useful across different system designs [25]-[27]. Also, the authors are not aware of any freely-accessible modeling tools or power system design software add-ons that account for both the harmonic and multi-state complexities of EV charges.

Our proposed solution treats EV charger models not as constant power elements, but considers their state of charge (SOC), which changes throughout the charging cycle. These charger models are not created by using a generalized circuit, as is popular in the current body of work, but uses analytical methods to map actual harmonic load data to a time-domain equivalent behavioral model of the non-linear impedance. This makes the load models responsive to the applied sinusoidal voltage of the system which, in the behavioral model, illustrates the effects, such as current, THD, and power flow, that the chargers have on distribution systems and their assets of interest. The discrete set of SOCs allows the user to analyze the system at various states in the charging cycle, while forgoing the calculationally-intensive process that would be required of a fully dynamic model.

As part of this work, a power system design tool was created, which is now freely available on the systemvision.com website. It is a free and collaborative environment where distribution system designers can layout, and analyze the response to, any system of interest, especially ones that include power system, three-phase, and EV charger components. The flexibility of these models also enables the user to examine existing, proposed, and retrofitted distribution systems that may include EV chargers. There is the important ability to examine chargers in aggregate as well as run different simulations for chargers coming on and off line at various points in their charging cycles. Based on the user's needs, specific aspects of power quality can be examined for a given system such as power flow, current content, THD, TDD, and load imbalance effects. This enables the user to not only utilize the tool to inform them in their design decisions, it can assist the designer in calculating values for transformers, conductors, and protection equipment.

\section{Methodology}

\section{A. Data Collection}

"Electric Avenue" was a collection of EV charging stations located on the Portland State University campus. It served as a research platform for studying the impacts of electric vehicles within the context of a city. For this research, PQ measurements were gathered from the electric vehicle chargers along Electric Avenue. From these data were calculated the THD of individual EV chargers and the TDD of the Electric Avenue service. The phase imbalance, parasitic loading, and other PQ issues observed during the course of the study were also noted. 
The site had five Level 2 chargers and two Level 3 chargers, donated by six different manufacturers. The Level 2 units are alternating current (AC), single phase, two-wire machines that, when attached to an EV manufactured with an SAE J1772 charging receptacle, replenish the EV's battery with a 4 to 20 $\mathrm{kW}$ input at $208 \mathrm{~V}$ [28]. Depending on the vehicle type, it can take up to 8 hours or more to fully replenish a depleted set of batteries. The power electronics that control the flow for the Level 2 type chargers are located within the vehicles themselves. The Level 3 charging units are three-phase designs that deliver power through a CHAdeMO standard receptacle. These are capable of delivering between 20 and $50 \mathrm{~kW}$ via direct current (DC) and can recharge an EV in as little as 30 minutes [29]. The power electronics that control the power for the Level 3 chargers are located off-board the vehicle, within the charging unit itself.

A Tektronix PA4000 three-phase power analyzer was use to collect data. CTs and voltage clamps were connected to the branch circuits of each of three chargers within the service panel. Data were recorded during real-time charging events. The PA4000 reports magnitude and phase angle of current and voltage for all odd harmonics up to the twenty-first. The reporting rate is $0.5 \mathrm{samples} / \mathrm{second}$. The site was monitored during data collection, so charging events were correlated with EV model types for each event that occurred.

The data were exported from the PA4000 using the Tektronix PWRVIEW Analysis software to Excel .xlsx files via a laptop at the site. The data sets grew large enough to exceed the processing capabilities of the laptop in less time than a full vehicle charging cycle. To compensate for this, sets of data were exported approximately every 30 minutes and later stitched together, using a desktop system, into larger .xlsx files. Since information stored in this format is highly flexible and, therefor, can be utilized by many different types of software, it proved to be valuable for use in analysis and modeling applications.

\section{B. Modeling}

Several power system simulation environments were considered for this work, but none offered the ability to model these multi-state, harmonic loads. Rather, a modeling tool was adopted that uses VHDL-AMS, an IEEE standard hardware modeling format [30]. IEEE Standard 1076.1 (VHDL-AMS) is a super-set of IEEE Standard 1076, a digital system hardware description language. VHDL-AMS provides analog and mixed-signal (AMS) modeling capabilities. SystemVision ${ }^{1}$, a modeling, simulation, and design analysis platform, provides the ability to create custom, highly configurable models of analog power distribution system elements, such as transformers, transmission lines, circuit breakers, and generators. The simulation environment is available for use in a free and easily accessible on-line simulation environment, where users can configure a model of their own distribution system using generic open-source models of fundamental power system equipment. The authors have developed a blog that demonstrates how power systems engineers can use the EV charger model. ${ }^{2}$

\footnotetext{
${ }^{1}$ SystemVision by Mentor, A Siemens Business. www.systemvision.com

${ }^{2}$ EV Charger Model Blog: www.systemvision.com/blog/comprehensivepower-system-modeling-and-simulation-july-31-2014
}

1) EV Charger: In order to model an EV charger, a multistate model must present an impedance that is a function of its charging cycle. For example, as a battery approaches full charge, the charge controller adjusts the power electronics firing angle to begin "trickle-charging" the battery, thereby shifting to a different charging state. This causes the controller to decrease the charge current in order to not overcharge the battery. As the firing sequence adjusts to start the tricklecharge state, the relative magnitudes of the harmonics increase, compared to the magnitude of the fundamental, even though the RMS current magnitude decreases. These periods of high harmonic loading are of interest because of the potential negative affects on distribution assets.

Data from several distinct states throughout the charging cycle are used to represent the time-varying loads. This multistate EV charger model allows for better representation of a distribution feeder that has multiple EV chargers attached to it. For instance, TDD may be monitored as multiple chargers come on line stochastically and proceed through their charging states. These models were created as active rather than passive components in order to accurately simulate their non-linear impedance in response to the applied voltage on the system.

In order to simulate the chargers at representative points throughout their cycles, current magnitude and phase angle data, through the fifth harmonic, were used to construct the EV charger models. A higher order model, through the ninth harmonic, was investigated, and gave accurate results. But, simulation speed and convergence robustness was reduced. As such, the fifth harmonic version was deemed sufficient and was used for this study. Even harmonics are neglected since they contribute to THD when there is t-axis asymmetry. This asymmetry across the t-axis is not present in EV chargers that are functioning properly. Current magnitudes and phase angles were selected from representative ranges of time throughout the charging cycle and represent the changing levels of THD presented to the system by the charger.

2) Analytical Solution: The modeling method ensures the load is responsive to the applied terminal voltage. This ensures the load behaves as a non-linear impedance rather than as a current source. The voltage across the terminals of the load is read, time-domain mathematical processing of the voltage is performed, and the result is the value of the current that flows through the device terminals. Figure 2 shows a harmonic load model schematic. Equation 4 described the time-domain current response to an applied voltage. The terminal voltage, $v(t)$, is scaled by the inverse of the peak voltage $\left(1 / V_{p}\right)$ of the sinusoidal stimulus at which the original load was calibrated, thereby normalizing the voltage, $v_{n}(t)$. To generate the fundamental current, $v(t)$ is integrated, differentiated, and then scaled by gain coefficients $k_{1 i}, k_{1 d}$, and $k_{1}$. To obtain the third harmonic current, the voltage is cubed, then integrated, differentiated, and scaled by gain coefficients $k_{3 i}, k_{3 d}$, and, $k_{3}$. The process is repeated for the fifth harmonic. The resulting currents are then summed, which then flow between the model terminals, $p 1$ and $p 2$.

The function results in a non-linear characteristic containing multiple harmonic components. The gain coefficients can be selected to fit the desired spectral content. Only four coefficients are actually needed to match the desired magnitude and phase of these two harmonics. But having 6 allows the 


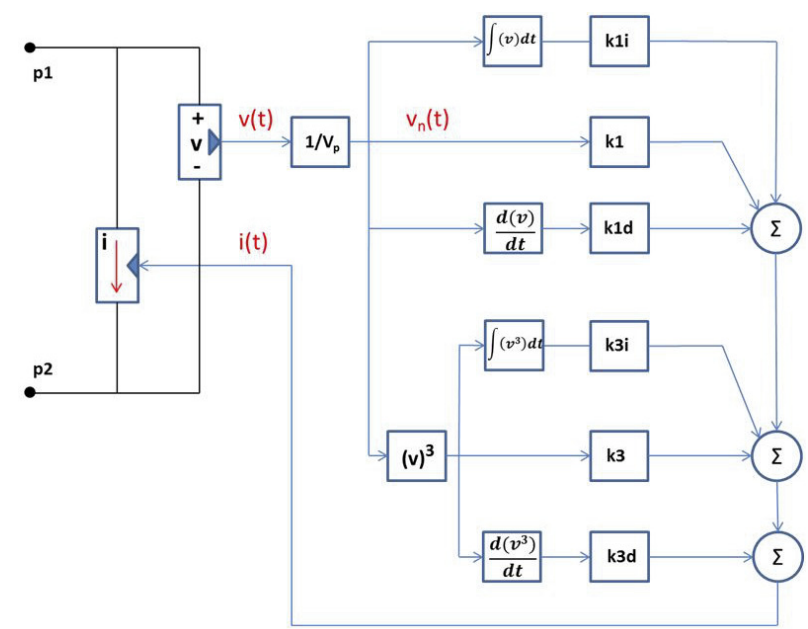

Fig. 2: A schematic of a simplified version of the AC harmonic load model, showing the conditioning of the $1^{\text {st }}$ and $3^{r d}$ harmonics.

selection algorithm to assign two of the set $\left\{k_{1 i}, k_{1 d}, k_{3 i} k_{3 d}\right\}$ to zero, so the remaining non-zero gains are positive. This is important for the reactive current contributors, for simulation stability.

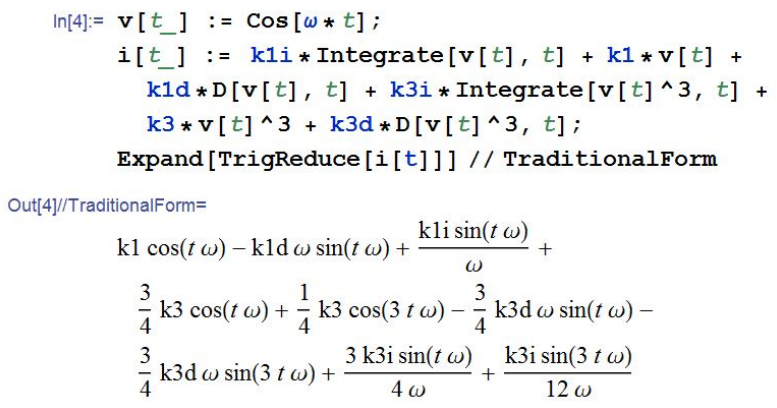

Fig. 3: Analysis of the model's time-domain current response, using Mathematica, to an applied sinusoidal voltage at frequency $\omega$.

$$
\begin{gathered}
v(t)=\frac{V}{V_{p}} \cos (\omega t) \\
i(t)=k_{1} v(t)+k_{1 i} \int v(t) d t+k_{1 d} \frac{d v(t)}{d t}+ \\
k_{3} v^{3}(t)+k_{3 i} \int v^{3}(t) d t+k_{3 d} \frac{d v^{3}(t)}{d t} \ldots
\end{gathered}
$$

The applied voltage amplitude and frequency must be approximately equal to the voltage at which the harmonics were measured. For that stimulus, this behavioral model accurately represents the complex, non-linear load from which the harmonic data were obtained. But, the model cannot predict the harmonic response under other stimulus conditions. The model provides the correct load current regardless of the stimulus voltage phase angle. The current depends on the applied voltage since it is not modeled as an independent current source. This allows multiple chargers to come on and offline at any point along the time axis without needing to be synchronized with the voltage phase.

\section{IEEE 13 Node Test Feeder}

The IEEE 13 node test feeder model is a standard model used for research and engineering [31]. This model was used to validate the VHDL-AMS modeling environment. The purpose of the model is to provide a common distribution model that can be used by engineers and researchers. A specification of voltage profiles and radial power flows is provided within the IEEE 13 node specification against which users may validate their models. All of the papers and spreadsheets with system values are maintained by the IEEE PES Distribution Test Feeder Working Group [32]. For the case of validating the use of VHDL-AMS for use in this research, the IEEE-13 node specification is immensely valuable. Hernandez and Canesin used the IEEE-13 node test feeder to validate their VHDLAMS environment as part of their development of a real-time digital simulator for electrical power distribution feeders [33]. Their paper illustrates the successful use of the IEEE 13 node test feeder as a verification tool for the VHDL-AMS language.

\section{ANALYSIS \& RESULTS}

The following section discusses the validation of the IEEE 13 node test feeder within the SystemVision environment. This is followed by analysis of the raw data current signals for the EV chargers versus the charger current signals, as modeled and simulated in the SystemVision environment, as well as TDD resulting from the charger models when connected to a three-phase system within the simulation environment. Finally, an analysis of the behavior of the IEEE 13 node test feeder, as the EV charger loads are added, is presented.

\section{A. EV Chargers on Three-Phase Systems}

For each of the charger models, Level 2 and Level 3, the raw data were first processed in Matlab in order to show the original waveforms. Next, the VHDL-AMS charger models were simulated in SystemVision. Then, comparisons were made between the two.

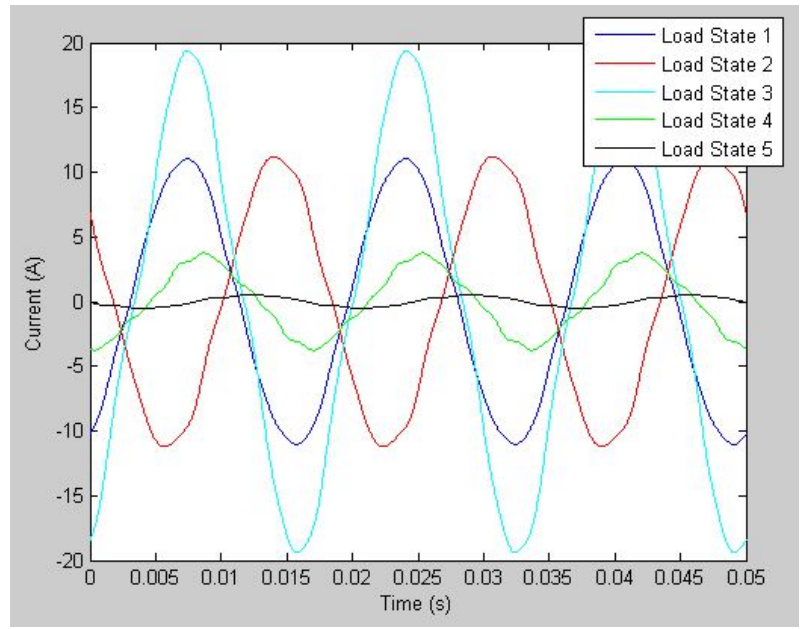

Fig. 4: Overlay of currents of the Level 2 chargers at each of the five charging states created in Matlab from raw data. 


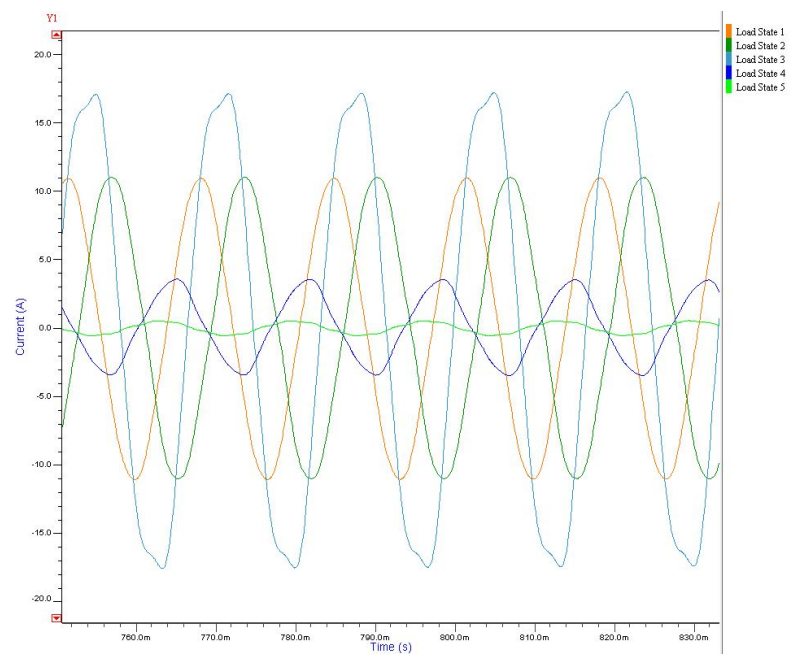

Fig. 5: Overlay of currents of the Level 2 chargers at each of the five charging states simulated in SystemVision from VHDL-AMS model.

1) Level 2 Charger: Current waveforms as produced in Matlab from the raw data for the Level 2 charger are shown in Figure 4. Each of the waveforms represents one of the five charging states.

The current waveforms as produced by a simulation run for the Level 2 charger model in SystemVision are shown in Figure 5. These waveforms are at least one second after the charger begins its simulation run. This is done to get through the transient start-up period that occurs at the beginning of each of the simulation runs. The magnitude of the currents correlate with the current profiles produced in Matlab. There are some slight variations in the waveform distortions that can be attributed to only the $3^{r d}$ and $5^{t h}$ harmonics being included in the model while all of the harmonics recorded during data collection are included in the Matlab waveforms.

2) Level 3 Charger: The VHDL-AMS model of the Level 3 charger is similar to that of the Level 2 charger. The same analytical and behavioral techniques as for the Level 2 charger are used in conjunction with the current magnitude and phase data collected for the Level 3 charger. Then, three of the models are linked together in a grounded wye configuration in order to simulate one charger. The state of charge for each of the three components that make up the model should be set to the same state or erroneous results will be produced. When connected to a three-phase distribution system, the currents for each of the components will be $120^{\circ}$ out of phase with one another due to the phase separation of the source voltages.

Figure 6 illustrates the waveforms for each of the five charging states created in Matlab from the raw data. Figure 7 shows the same set of waveforms as simulated from the Level 3 charger model in SystemVision. Again, the current magnitudes correspond. Also, similar to the Level 2 case, more distortion can be seen in the Matlab output since all harmonics were included in that data set. In the case of the Level 3 charger, a reference angle of zero was used for each of the fundamantals for each charge state. The $3^{r d}$ and $5^{\text {th }}$ harmonics for each were then offset by their respective values. While this causes the entire waveform to be shifted along the t-axis, it does not change the effect that the harmonics of the charger have on the system under simulation.

\section{B. Validation of the IEEE 13 Node Test Feeder}

Loads on the IEEE 13 node test feeder consist of threephase balanced and unbalanced loads and single-phase loads. The small and relatively highly-loaded system has a system voltage of $4.16 \mathrm{kV}$, a very common distribution voltage. It typically presents no convergence problems. The steady-state voltage profiles and radial power flows are provided in the IEEE 13 node test feeder specification. The VHDL-AMS model differs from the IEEE specification by less than $0.5 \%$, indicating validation of the VHDL-AMS model.

The system response of most interest is the one where many chargers are connected to a distribution system and are set to various charging states such that the aggregate effect on the system can be observed. For the IEEE 13 node test feeder, the constant power load at node 645 was incrementally replaced by EV charger loads. Each time the percentage of loading from the EV charger was increased, the currents at the 645, 646, and 633 nodes were recorded. These nodes include the node at the chargers, one node away from the chargers, and two nodes away from the chargers, respectively. An FFT analysis was done on each of these waveforms in order to assess the harmonic content of each.

Table I shows the TDD as calculated for the system at each of the nodes. The current distortion limits allowed as defined by IEEE Standard 519-1992 for the system are based on the ratio of the available short circuit current to the maximum demand load current. Since the short circuit current for this system is so high compared to the maximum demand load current, current distortion levels of up to $10 \%$ are allowed through the $11^{\text {th }}$ harmonic. Table II shows harmonic current as a function of charging state for the Level $2 \mathrm{EV}$ charger model, demonstrating that THD increases as charge state advances.

\section{DISCUSSION}

Validation of the bus voltage profiles and the radial power flows for the VHDL-AMS model, compared to the IEEE 13 node test feeder specifications, showed model correlation within less than $0.5 \%$. This indicates sufficient construction of the model components and the feeder schematic within the

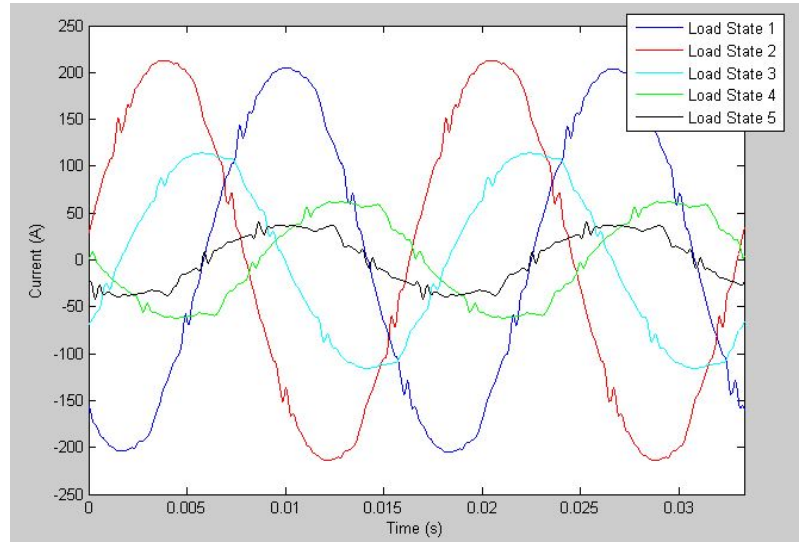

Fig. 6: Overlay of currents of the Level 3 chargers at each of the five charging states created in Matlab from raw data. 
TABLE I: TDD for three nodes in the IEEE 13 node test feeder when EV charger loads are incrementally added at node 645 .

\begin{tabular}{cccc}
\multicolumn{4}{c}{ Total Demand Distortion (TDD) } \\
\hline \hline EV \% of Load & Node 645 & Node 646 & Node 633 \\
\hline 10 & $0 \%$ & $0 \%$ & $0 \%$ \\
20 & $0 \%$ & $0 \%$ & $0 \%$ \\
30 & $1 \%$ & $0 \%$ & $0 \%$ \\
40 & $1 \%$ & $1 \%$ & $0 \%$ \\
50 & $2 \%$ & $1 \%$ & $0 \%$ \\
60 & $3 \%$ & $2 \%$ & $1 \%$ \\
70 & $4 \%$ & $3 \%$ & $1 \%$ \\
80 & $5 \%$ & $3 \%$ & $1 \%$ \\
90 & $5 \%$ & $4 \%$ & $2 \%$ \\
100 & $6 \%$ & $5 \%$ & $2 \%$
\end{tabular}

TABLE II: Harmonic current as a function of charging state: Level 2 EV charger model, showing THD increases as charge state advances.

\begin{tabular}{cccccc}
\multicolumn{5}{c}{ Harmonic Current Magnitude (A) } \\
\hline \hline Load State & Fund & $3^{\text {rd }}$ & $5^{t h}$ & $\mathrm{I}_{R M S}$ & THD \\
\hline 1 & 27.4 & 3.92 & 1.31 & 27.7 & $14.9 \%$ \\
2 & 20.2 & 4.15 & 1.26 & 20.6 & $21.1 \%$ \\
3 & 13.2 & 3.64 & 0.80 & 13.7 & $27.2 \%$
\end{tabular}

VHDL-AMS environment. Deviations in all three did occur more often in the nodes that were farther away from the substation in the model. This is most likely a calculation error. As the lengths of the lines increase, the magnitude of the resistance and impedance grows larger and any rounding that was used to enter their values will start to effect the final calculation of power flow on the system.

The results for the power flow analysis on the IEEE 13 node test feeder when EV chargers were connected to the feeder show the TDD affect to be limited to nearby nodes; the distortion effects that the chargers caused were localized around the immediate area where they were installed. The TDD at all three nodes was low, regardless of EV loading or charge state. The IEEE 13 node test feeder is a stiff feeder, and as such it is not prone to harmonics propagating far from their source. The TDD impacts of the EV chargers on a smaller and less stiff distribution feeder could be greater.

Other researchers have shown that THD is usually low

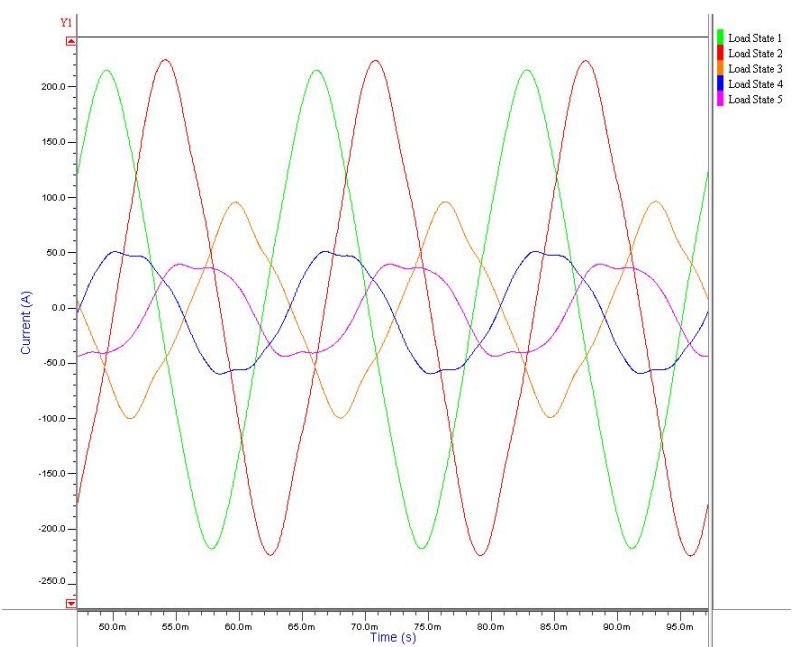

Fig. 7: Overlay of currents of the Level 3 chargers at each of the five charging states simulated in SystemVision from VHDL-AMS model.

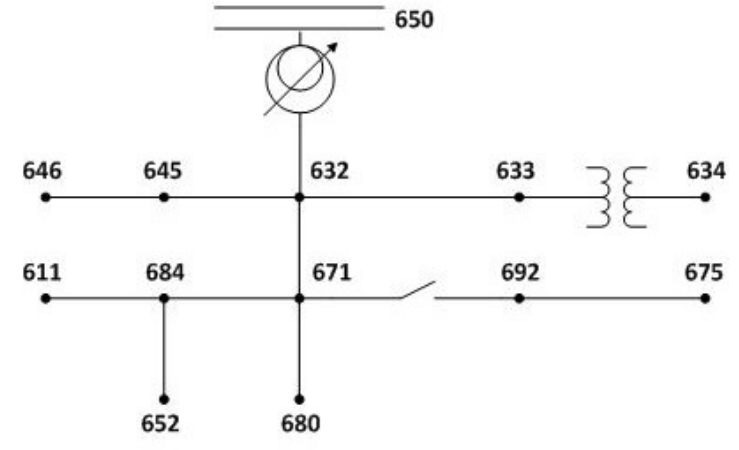

Fig. 8: One-line diagram of IEEE 13 node test feeder, with nodes labeled.

during EV charging [34]-[36]. However, TDD is a more relevant metric for the harmonic content within a distribution feeder. THD is a measure of the harmonic distortion from a single load, hence the use of $I_{1}$ in the definition denominator. By using $I_{L}$ in the denominator instead, and considering the harmonics in the feeder, TDD quantifies the harmonics resulting from all loads with respect to the loading on the feeder.

\section{CONCLUSION}

Presented is a solution for modeling non-linear EV charger loads in various charge states. These models were created based on actual data from EV chargers at public charging stations. The IEEE 13 node test feeder was used to validate the VHDL-AMS simulation environment within which the models were created. Further, the EV charger models were simulated within the IEEE 13 node test feeder in order to analyze their effects. Also, a cloud-based tool for distribution engineers was created. ${ }^{3}$ This tool enables engineers to run simulations that include EV charger models in order to analyze the effects that they may have on new or existing distribution systems.

The installation rate of new EV chargers within power distribution systems will continue to increase into the foreseeable future. Models of EV chargers will help distribution designers predict the impacts that these loads may have on distribution assets. When distribution engineers are able to make these predictions during the early planning phases of projects, they are better able to mitigate the negative impacts that EV chargers may have on power system assets. Minimizing these impacts facilitates the interconnection of EV chargers within a utility's balancing area. This in turn impacts the adoption of EVs, which will be vital to developing a low-carbon transportation future.

\section{ACKNOWLEDGMENT}

The authors would like to thank Portland General Electric for providing access to the Portland State University Electric Avenue feeder service panel, Tektronix for the donation of PA4000 power analyzers and Mentor, A Siemens Business, for technical support with SystemVision.

\footnotetext{
${ }^{3}$ SystemVision $3 \phi$ EV Load model: sysvis.ioev_charging_harmonics
} 


\section{REFERENCES}

[1] S. Habib, M. M. Khan, F. Abbas, L. Sang, M. U. Shahid, and H. Tang, "A comprehensive study of implemented international standards, technical challenges, impacts and prospects for electric vehicles," IEEE Access, vol. 6, pp. 13866-13890, 2018.

[2] "Alternative Fuels Data Center, U.S. Department of Energy, Office of Energy Efficiency \& Renewable Energy," (Accessed: June 22, 2018).

[3] I. Hunter, "Power quality issues: a distribution company perspective," Power Eng. J. , vol. 15, no. 2, pp. 75-80, 2001.

[4] M. C. Falvo, D. Sbordone, I. S. Bayram, and M. Devetsikiotis, "EV charging stations and modes: International standards," in 2014 International Symposium on Power Electronics, Electrical Drives, Automation and Motion, pp. 1134-1139, June 2014.

[5] R. B. Bass, M. Donnelly, and N. Zimmerman, "Time-varying modeling of electric vehicle chargers," in 2014 IEEE Conference on Technologies for Sustainability (SusTech), pp. 245-249, July 2014.

[6] "IEEE recommended practice for establishing liquid-filled and drytype power and distribution transformer capability when supplying nonsinusoidal load currents," IEEE Std C57.110-2008, Aug 2008.

[7] "IEEE recommended practices and requirements for harmonic control in electrical power systems," IEEE Std 519-1992, 1993.

[8] D. Said and K. Nor, "Effects of harmonics on distribution transformers," in Australasian Universities Power Engineering Conf., pp. 1-5, 2008.

[9] A. Elmoudi, M. Lehtonen, and H. Nordman, "Effect of harmonics on transformers loss of life," in Electrical Insulation, 2006. Conf. Proc. 2006 IEEE Int. Symposium on, pp. 408-411, 2006.

[10] P. Dahono, R. Widjaya, Syafrudin, and Qamaruzzaman, "A practical approach to minimize the zero-sequence current harmonics in power distribution systems," in Power Conv. Conf., vol. 2, pp. 683-686, 1997.

[11] A. K. Hiranandani, "Effects of harmonics on the current carrying capacity of insulated power cables used in three phase electrical power distribution systems," in 18th Int. Conf. and Expo. on Electricity Distribution, pp. 1-5, 2005.

[12] "IEEE standard definitions for the measurement of electric power quantities under sinusoidal, nonsinusoidal, balanced, or unbalanced conditions," IEEE Std 1459-2010, pp. 1-50, 2010.

[13] A. Emanuel, Power definitions and the physical mechanism of power flow. Chichester, West Sussex: Wiley, 2010.

[14] A. Cataliotti, V. Cosentino, D. D. Cara, and G. Tin, "IEEE std 1459 power quantities ratio approaches for simplified harmonic emissions assessment," in 2018 18th International Conference on Harmonics and Quality of Power (ICHQP), pp. 1-6, May 2018.

[15] G. Putrus, P. Suwanapingkarl, D. Johnston, E. Bentley, and M. Narayana, "Impact of electric vehicles on power distribution networks," in IEEE Vehicle Power \& Prop. Conf., pp. 827-831, 2009.

[16] O. Ceylan, S. Paudyal, S. Dahal, and N. R. Karki, "Assessment of harmonic distortion on distribution feeders with electric vehicles and residential PVs," in 2017 7th International Conference on Power Systems (ICPS), pp. 621-626, Dec 2017.

[17] A. A. Malano, S. Mller, J. Meyer, and S. Bachmann, "Harmonic interaction of electric vehicle chargers in a central charging infrastructure," in 2016 17th International Conference on Harmonics and Quality of Power (ICHQP), pp. 367-372, Oct 2016.

[18] T. Hoevenaars, K. LeDoux, and M. Colosino, "Interpreting IEEE std 519 and meeting its harmonic limits in VFD applications," in IEEE Industry Applications Society 50th Annu. Petroleum and Chemical Industry Conf., pp. 145-150, 2003.

[19] J. Sexauer, K. McBee, and K. Bloch, "Applications of probability model to analyze the effects of electric vehicle chargers on distribution transformers," IEEE Trans. Power Sys., vol. 28, pp. 847-854, May 2013.

[20] A. Dubey, S. Santoso, and M. Cloud, "Average-value model for plug-in hybrid electric vehicle battery chargers," in IEEE PES General Meeting, pp. 1-8, July 2012.

[21] S. Zhao, S. Haghbin, O. Wallmark, M. Leksell, S. Lundmark, and O. Carlson, "Transient modeling of an integrated charger for a plug-in hybrid electric vehicle," in 14th European Conf. on Power Electronics and Applications, pp. 1-10, Aug 2011.
[22] A. Haidar and K. Muttaqi, "Behavioral characterization of electric vehicle charging loads in a distribution power grid through modeling of battery chargers," in IEEE Ind. Appl. Soc., pp. 1-8, Oct 2014.

[23] L. Chen and Y. Zhang, "Model and harmonic control strategy of electric vehicle chargers connected with the power system," in IEEE Power Engineering and Automation Conf., vol. 2, pp. 524-528, Sept 2011.

[24] T. McDermott, R. Juchem, and D. Devarajan, "Distribution feeder and induction motor modeling with VHDL-AMS," in IEEE PES Transmission and Distribution Conf. and Expo., pp. 141-146, May 2006.

[25] F. Moller, J. Meyer, and P. Schegner, "Load model of electric vehicles chargers for load flow and unbalance studies," in Electric Power Quality and Supply Reliability Conf., pp. 9-14, June 2014.

[26] A. Collin, S. Djokic, H. Thomas, and J. Meyer, "Modelling of electric vehicle chargers for power system analysis," in 11th Int. Conf. on Electrical Power Quality and Utilisation, pp. 1-6, Oct 2011.

[27] R. Horton, J. Taylor, A. Maitra, and J. Halliwell, "A time-domain model of a plug-in electric vehicle battery charger," in IEEE PES Transmission and Distribution Conf. and Exposition, pp. 1-5, May 2012.

[28] T. Bohn and H. Chaudhry, "Overview of SAE standards for plug-in electric vehicle," in IEEE PES Innov. Smart Grid Tech., pp. 1-7, 2012.

[29] M. Yilmaz and P. Krein, "Review of charging power levels and infrastructure for plug-in electric and hybrid vehicles," in IEEE Int. Electric Vehicle Conf., pp. 1-8, 2012.

[30] P. Ashenden, G. Peterson, and D. Teegarden, The System Designer's Guide to VHDL-AMS: Analog, Mixed-Signal, and Mixed-Technology Modeling. Systems on Silicon, Elsevier Science, 2002.

[31] W. Kersting, "Radial distribution test feeders," in IEEE PES Winter Meeting, vol. 2, pp. 908-912 vol.2, 2001.

[32] "IEEE PES Distribution System Analysis Subcommittee Distribution Test Feeder Working Group," August 2013.

[33] F. Ibarra Hernandez and C. Canesin, "Electrical power distribution system modeling with VHDL-AMS for the construction of a real-time digital simulator using FPGAS devices," in 10th IEEE/IAS Int. Conf. on Industry Applications, pp. 1-7, Nov 2012.

[34] J. D. Watson and N. R. Watson, "Impact of electric vehicle chargers on harmonic levels in New Zealand," in 2017 IEEE Innovative Smart Grid Technologies - Asia (ISGT-Asia), pp. 1-6, Dec 2017.

[35] A. Ul-Haq, A. Perwaiz, M. Azhar, and S. U. Awan, "Harmonic distortion in distribution system due to single-phase electric vehicle charging," in 2018 2nd International Conference on Green Energy and Applications (ICGEA), pp. 205-209, March 2018.

[36] M. D. Paolo, "Analysis of harmonic impact of electric vehicle charging on the electric power grid, based on smart grid regional demonstration project - Los Angeles," in 2017 IEEE Green Energy and Smart Systems Conference (IGESSC), pp. 1-5, Nov 2017. 